\title{
A depression before a bump in the highest energy cosmic ray spectrum
}

\author{
L. A. Anchordoqui, M. T. Dova and L. N. Epele \\ Departamento de Física, Universidad Nacional de La Plata \\ C.C. 67, (1900) La Plata \\ Argentina \\ J. D. Swain \\ Department of Physics, Northeastern University \\ Boston, Massachusetts, 02115 \\ $U S A$

\begin{abstract}
We re-examine the interaction of ultra high energy nuclei with the microwave background radiation. We find that the giant dipole resonance leaves a new signature in the differential energy spectrum of iron sources located around $3 \mathrm{Mpc}$ : A depression before the bump which is followed by the expected cutoff.

PACS number(s): 96.40, 13.85.T, 98.70.S, 98.70.V
\end{abstract}

\section{INTRODUCTION}

In 1966 Greisen, Zatsepin and Kuz'min [1.22 noted that the microwave background radiation (MBR) makes the universe opaque to cosmic rays of sufficiently high energy, yielding a steep drop in the energy cosmic ray spectrum at approximately $5 \times 10^{19} \mathrm{eV}$ (GZK cutoff). More recently, a fresh interest in the topic has been rekindled since several extensive air showers have been observed which imply the arrival of cosmic rays with energies above $10^{20} \mathrm{eV}$. In particular, the Akeno Giant Air Shower Array (AGASA) experiment recorded an event with energy $1.7-2.6 \times 10^{20} \mathrm{eV}$ [3, 目, the Fly's Eye experiment reported the highest energy cosmic ray event ever detected

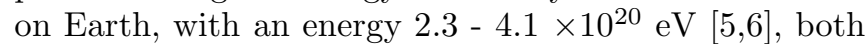
events being well above the GZK cutoff. Deepening the mystery, the identification of the primary particle in these showers is still uncertain. On the one hand, the Fly's Eye group claims that there is evidence of a transition from a spectrum dominated by heavy nuclei to one of a predominantly light composition [5], while on the other hand, it has also been suggested that a medium mass nucleus also fits the shower profile of the highest energy Fly's Eye event [7]. In addition, there is an unexpected energy gap before these events. Although heavy nuclei can be accelerated to high terminal energies by "bottom up" mechanisms, one should note that, for energies above $100 \mathrm{EeV}$ the range of the corresponding sources is limited to a few Mpc 11. Sigl and co-workers [8] have analysed the structure of the high energy end of the cosmic ray spectrum. They found that most "bottom up" models can be ruled out except for those involving a nearby source, which is consistent with data at the $1 \sigma$ level. Their argument for this is that a nearby source can account for the ultrahigh energy events but would also produce events in the apparent gap in data obtained to date. In this direction, Elbert and Sommers have suggested that the highest energy event recorded by Fly's Eye, could have been accelerated in the neighborhood of M82, which is around 3
Mpc away [9.10]. In relation to the aforementioned possibilities, we have re-examined the interaction of ultrahigh energy nuclei with the microwave background radiation and we have found a new feature in the ultrahigh energy cosmic ray spectrum from iron sources located around 3 Mpc which forms the motivation for the present article.

\section{ENERGY ATTENUATION LENGTH OF ULTRAHIGH ENERGY NUCLEI}

The energy losses that extremely high energy nuclei suffer during their trip to the Earth are due to their interaction with the low energy photons of the MBR which they see as highly blue-shifted. The interaction with other radiation backgrounds (optical and infrared) can be safely neglected for nuclei with Lorentz factors above $2 \times 10^{9}$. Although the interactions of extremely high energy nuclei with the relic photons lead to step-by-step energy loss (which needs to be included in a transport equation as a collision integral), in what follows we use the continuous energy loss approximation assuming straight line propagation which is reasonable for the energies and distances under consideration in this paper. The relevant mechanisms for energy losses are photodisintegration and hadron photoproduction (which has a threshold energy of $\approx 145 \mathrm{MeV}$, equivalent to a Lorentz factor of $10^{11}$, above the range treated in this article) [12].

The disintegration rate of a nucleus of mass $A$ with the subsequent production of $i$ nucleons is given by the expression [13],

$$
R_{A i}=\frac{1}{2 \Gamma^{2}} \int_{0}^{\infty} d w \frac{n(w)}{w^{2}} \int_{0}^{2 \Gamma w} d w_{r} w_{r} \sigma_{A i}\left(w_{r}\right)
$$

where $n(w)$ is the density of photons with energy $w$ in the system of reference in which the microwave background is at $3 \mathrm{~K}$ and $w_{r}$ is the energy of the photons in the rest frame of the nucleus. As usual, $\Gamma$ is the Lorentz factor and $\sigma_{A i}$ is the cross section for the interaction. Using 


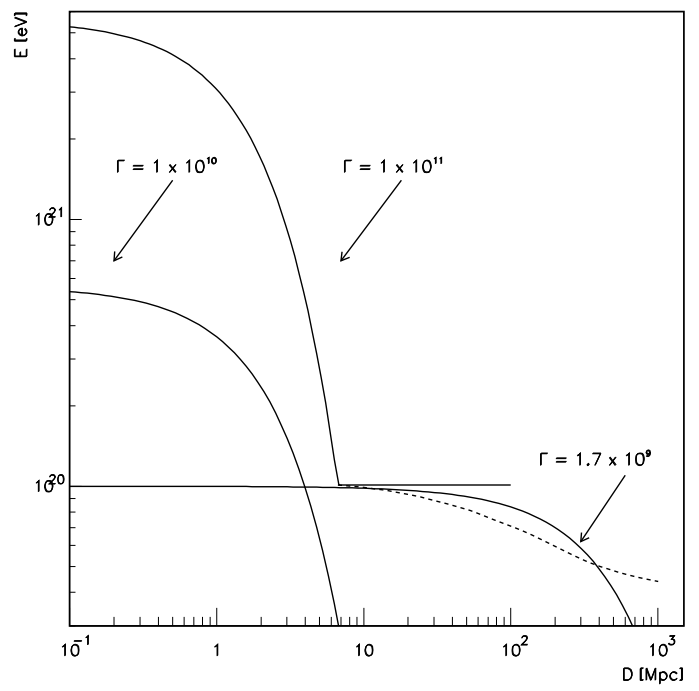

FIG. 1. Energy of the surviving nuclei vs. propagation distance. It is also included the energy attenuation length of the surviving nucleon (dot line).

the expressions for the cross section fitted by Puget et al. [14], it is possible to work out an analytical solution for the nuclear disintegration rates [15]. After summating them over all the possible channels for a given number of nucleons one obtains the effective nucleon loss rate. The effective ${ }^{56} \mathrm{Fe}$ nucleon loss rate obtained after carrying out these straightforward but rather lengthy steps can be parametrized by,

$$
R(\Gamma)=3.25 \times 10^{-6} \Gamma^{-0.643} \exp \left(-2.15 \times 10^{10} / \Gamma\right) \mathrm{s}^{-1}
$$

if $\Gamma \in\left[1 . \times 10^{9}, 3.68 \times 10^{10}\right]$, and

$$
R(\Gamma)=1.59 \times 10^{-12} \Gamma^{-0.0698} \mathrm{~s}^{-1}
$$

if $\Gamma \in\left[3.68 \times 10^{10}, 1 . \times 10^{11}\right]$. It is noteworthy that knowledge of the iron effective nucleons loss rate alone is enough to obtain the corresponding value of $R$ for any other nuclei [14].

The emission of nucleons is isotropic in the rest frame of the nucleus, and so the averaged fractional energy loss results equal the fractional loss in mass number of the nucleus, viz., the Lorentz factor is conserved. The relation which determines the attenuation length for energy is then, assuming an initial iron nucleus,

$$
E=E_{g} e^{-R(\Gamma) t / 56}
$$

where $E_{g}$ denotes the energy with which the nuclei were emitted from the source, and $\Gamma=E_{g} / 56$.

In Fig. 1 we have plotted the total energy of the heaviest surviving fragment as a function of the distance for initial iron nuclei. Note that the values obtained here

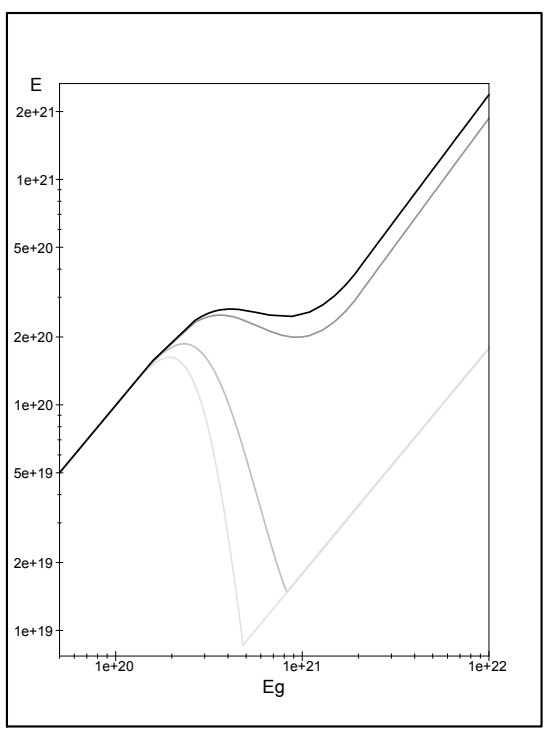

FIG. 2. Relation between the injection energy of an iron nucleus and the final energy of the photodisintegrated nucleus for different values of the propagation distance (from grey to black $20 \mathrm{Mpc}, 10 \mathrm{Mpc}, 3.5 \mathrm{Mpc}, 3 \mathrm{Mpc}$ ).

are consistent with the ones obtained by Cronin using Monte Carlo simulation 11. One can see that nuclei with Lorentz factors above $10^{10}$ cannot survive for more than $10 \mathrm{Mpc} 16$.

In Fig. 2 the relation between the injection energy and the energy at a time $t$ for different propagation distances is shown. The graph indicates that the final energy of the nucleus is not a monotonic function. It has a maximum at a critical energy and then decreases to a minimum before rising again as $\Gamma$ rises, as was first pointed out by Puget et al. 114. The fact that the energy $E$ is a multivaluated function of $E_{g}$ leads to a pile-up in the energy spectrum. Moreover, this behaviour enhances a hidden feature of the energy spectrum for sources located beyond 2.6 Mpc: A depression that preceeds a bump that would make the events at the end of the spectrum (just before the cutoff) around $50 \%$ more probable than those in the depressed region. To illustrate this, let us discuss the evolution of the differential energy spectrum of nuclei.

\section{MODIFICATION OF THE COSMIC RAY SPECTRUM}

The photodisintegration process results in the production of nucleons of ultrahigh energies with the same Lorentz factor of the parent nucleus. As a consequence, the total number of particles is not conserved during propagation. However, the solution of the problem becomes quite simple if we separately treat both the evolution of the heaviest fragment and those fragments corresponding to nucleons emitted from the travelling nuclei. 
The evolution of the differential spectrum of the surviving fragments is governed by a balance equation that takes into account the conservation of the total number of particles in the spectrum. Using the formalism presented by the authors in reference [17], and considering the case of a single source located at $t_{0}$ from the observer, with injection spectrum $Q\left(E_{g}, t\right)=\kappa E_{g}^{-\gamma} \delta\left(t-t_{0}\right)$, the number of particles with energy $E$ at time $t$ is given by,

$$
N(E, t) d E=\frac{\kappa E_{g}^{-\gamma+1}}{E} d E,
$$

with $E_{g}$ fixed by the constraint (3).

Let us now consider the evolution of nucleons generated by decays of nuclei during their propagation. For Lorentz factors less than $10^{11}$ and distances less than $100 \mathrm{Mpc}$ the energy with which the secondary nucleons are produced is approximately equal to the energy with which they are detected here on Earth. The number of nucleons with energy $E$ at time $t$ can be approximated by the product of the number of nucleons generated per nucleus and the number of nuclei emitted. When the nucleons are emitted with energies above $100 \mathrm{EeV}$ the losses by meson photoproduction start to become significant. However, these nucleons come from heavy nuclei with Lorentz factors $\Gamma>10^{11}$ which are completely disintegrated in distances of less than $10 \mathrm{Mpc}$. Given that the mean free path of the nucleons is about $\lambda_{n} \approx 10 \mathrm{Mpc}$, it is reasonable to define a characteristic time $\tau_{\Gamma}$ given by the moment in which the number of nucleons is reduced to $1 / e$ of its initial value $A_{0}$. In order to determine the modifications of the spectrum due to the losses which the nucleons suffer due to interactions with the relic photons, we assume that the iron nucleus emitted at $t=t_{0}$ is a travelling source which at the end of a time $\tau_{\Gamma}$ has emitted the 56 nucleons together. In this way the injection spectrum of nucleons $\left(\Gamma \approx 10^{11}\right)$ can be approximated by,

$$
q\left(E_{G}, t\right)=\kappa A_{0}^{-\gamma+1} E_{G}^{-\gamma} \delta\left(t-\tau_{\Gamma}\right),
$$

where $A_{0}$ is the mass of the initial nucleus and the energy with which the nucleons is generated is given by $E_{G}=$ $E_{g} / A_{0}$.

The number of nucleons with energy $E$ at time $t$ is given by,

$$
n(E, t) d E=\frac{\kappa A_{0}^{-\gamma+2} E_{G}^{-\gamma+1}}{E} d E
$$

and the relation between injection energy and the energy at time $t$ remains fixed by the relation, $A\left(t-\tau_{\Gamma}\right)-$ $\operatorname{Ei}(B / E)+\operatorname{Ei}\left(B / E_{G}\right)=0$, Ei being the exponential integral, and $A, B$ the parameters of the fractional energy loss of nucleons previously fitted by the authors [17].

The modification factor $\eta$, is defined as the ratio between the modified spectrum and the unmodified one. In Fig. 3 we plot the modification factors for the case of sources of iron nuclei (propagation distance $20 \mathrm{Mpc}$ ) together with the spectra of secondary nucleons. It is clear

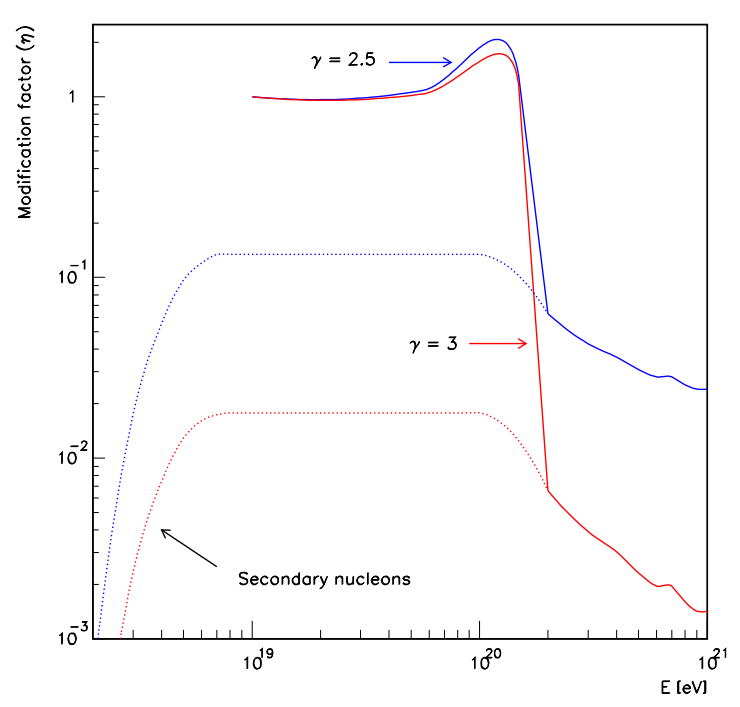

FIG. 3. Modification factors for sources of iron nuclei at 20 Mpc together with the spectra of secondary nucleons.

that the spectrum of secondary nucleons around the pileup is at least one order of magnitude less than the one of the surviving fragments. In Figures 4 and 5 we have plotted the modification factor for different propagation distances around $3 \mathrm{Mpc}$. They display a bump and a cutoff and, in addition, a depression before the bump. It is important to stress that the mechanism that produces the pile-up which can be seen in Figures 3,4 and 5 is completely different to the one that produces the bump in the case of nucleons.

In this last case, the photomeson production involves the creation of new particles that carry off energy yielding nucleons with energies ever closer to the photomeson production threshold. This mechanism, modulated by the fractional energy loss, is responsible for the bump in the spectrum. The cutoff is a consequence of the conservation of the number of particles together with the properties of the injection spectrum $\left(\int_{E_{\mathrm{th}}}^{\infty} E_{g}^{-\gamma} d E_{g}<\infty\right)$.

In the case of nuclei, since the Lorentz factor is conserved, the surviving fragments see the photons of the thermal background always at the same energy. Then, despite the fact that nuclei injected with energies over the photodisintegration threshold lose energy by losing mass, they never reach the threshold. The observed pile-up in the modification factors is due solely to the multivalued nature of the energy at time $t$ as a function of the injection energy: Nuclei injected with different energies can arrive with the same energy but with different masses.

It is clear that, except in the region of the pile-up, the modification factor $\eta$ is less than unity, since $\eta=$ $\left(E / E_{g}\right)^{\gamma-1}$. This assertion seems to be in contradiction with the conservation of particle number. Actually, the conservation of the Lorentz factor implies,

$$
\left.\kappa E_{g}^{-\gamma} d E_{g}\right|_{\Gamma}=\left.N(E, t) d E\right|_{\Gamma}
$$




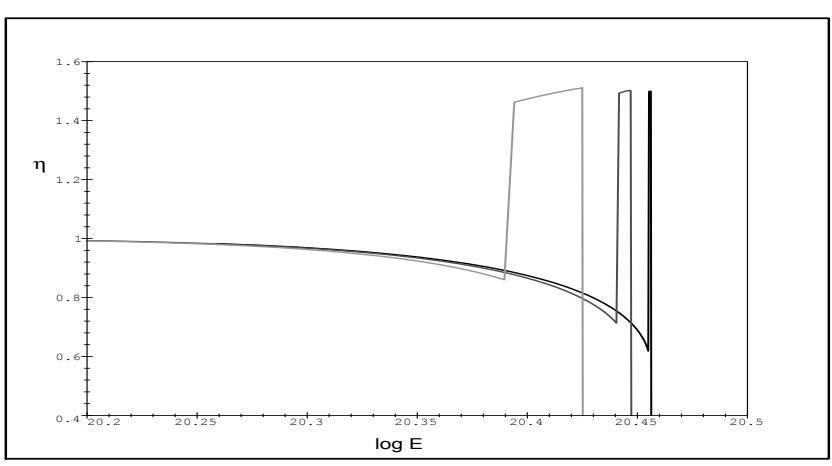

FIG. 4. Modification factor of single-source energy espectra for different values of propagation distance (from grey to black $3 \mathrm{Mpc}, 2.7 \mathrm{Mpc}$ and $2.6 \mathrm{Mpc}$ ) assuming a differential power law injection spectrum with spectral index $\gamma=2$.

in accord with the conservation of the number of particles in the spectrum. Moreover, the condition (7) completely determines the evolution of the energy spectrum of the surving fragments (41). Note that in order to compare the modified and unmodified spectra, with regard to conservation of particle number, one has to take into account that the corresponding energies are shifted. As follows from (7), the conservation of the number of particles in the spectrum is given by,

$$
\int_{E_{\mathrm{th}}}^{E_{\pi_{\mathrm{th}}}} N(E, t) d E=\int_{E_{g_{\mathrm{th}}}}^{E_{g \pi_{\mathrm{th}}}} \kappa E_{g}^{-\gamma} d E_{g}
$$

with $E_{\text {th }}$ and $E_{\pi_{\text {th }}}$ the threshold energies for photodisintegration, and photopion production processes respectively.

Let us now return to the analysis of Fig. 2 in relation to the depression in the spectrum. In the case of a nearby iron source, located around $3 \mathrm{Mpc}$, and for injection energies below the multivalued region of the function $E\left(E_{g}\right)$, $E$ is clearly less than $E_{g}$ and, as a consequence the depression in the modification factor is apparent. Then, despite the violence of the photodisintegration process via the giant dipole resonance, for nearby sources none of the injected nuclei are completely disintegrated yielding this unusual depression before the bump. For a flight distance of $3 \mathrm{Mpc}$, the composition of the arrival nuclei changes from $A=50$ (for $\Gamma \approx 10^{9}$ ) to $A=13$ (for $\Gamma \approx 10^{11}$ ). However, the most important variation takes place in the region of the bump, where $A$ runs from 48 to 13, being heavy nuclei of $A=33$ the most abundant. For propagation distances greater than $10 \mathrm{Mpc}$ one would expect just nucleons to arrive for injection energies above $9 \times 10^{20} \mathrm{eV}$. In this case the function becomes multivalued below the photodisintegration threshold and then there is no depression at all. For an iron source located at 3.5 Mpc, the depression in the spectrum is almost invisible $(\mathcal{O}(1 \%))$, in good agreement with the results previously obtained by Elbert and Sommers using Monte Carlo simulation 9.

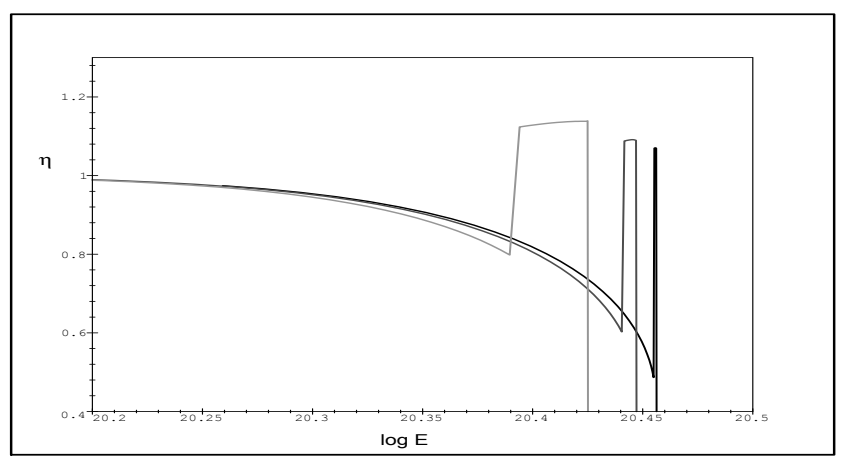

FIG. 5. Same as Fig. 4 with spectral index $\gamma=2.5$.

\section{CONCLUSIONS}

We have studied the interaction of ultra high energy nuclei with the MBR. We have presented a parametrization of the fractional energy loss for Lorentz factors up to $10^{11}$ that allows us to analyse the evolution of the energy spectrum for different nuclei sources. When considering an iron source located around $3 \mathrm{Mpc}$, the spectrum exhibits a depression before a bump not previously reported. In the light of this finding it is tempting to speculate whether the apparent gap in the existing data is due to the relative weight of the depression and the bump if a source of iron nuclei is responsible for the end of the cosmic ray spectrum. This speculation, if true, reclaims "botton up" models as a possible scenario for the origin of the highest energy cosmic rays. The limited statistics in the observed data make it impossible to resolve the question definitively at this time, and we are obliged to present this idea as a hypothesis to be tested by experiment.

The existence of a cutoff or a gap which might be present in the observed spectrum is of fundamental interest in cosmic ray physics, allowing stringent tests of existing models. The future Pierre Auger Project 18] should provide enough statistics for a final veredict on these open questions, and in particular on the ideas discussed in this paper.

\section{ACKNOWLEDGMENTS}

Special thanks go to Prof. James Cronin for stimulating discussions. This research was supported in part by CONICET and FONCYT. L.A.A. thanks FOMEC for financial support.

[1] K. Greisen, Phys. Rev. Lett. 16, 748 (1966). 
[2] G. T. Zatsepin and V. A. Kuz'min, Pis'ma Zh. Eksp. Teor. Fiz. 4, 114 (1966).

[3] S. Yoshida et al., Astropart. Phys. 3, 105 (1995).

[4] N. Hashashida et al., Phys. Rev. Lett. 73, 3491 (1994).

[5] D. J. Bird et al., Phys. Rev. Lett. 71, 3401 (1993).

[6] D. J. Bird et al., Ap. J. 441, 144 (1995).

[7] F. Halzen, R. A. Vazquez, T. Stanev and V. P. Vankov, Astropart. Phys. 3, 151 (1995).

[8] G. Sigl, S. Lee, D. N. Schramm, and P. Bhattacharjee, Science 270, 1977 (1995).

[9] J. W. Elbert and P. Sommers, Ap. J. 441, 151 (1995).

[10] To be more precise, the distance from the Earth to M82 belongs to the interval 2 to $3.5 \mathrm{Mpc}$, depending on the value of the Hubble constant. The reader is referred to S. Weinberg, Gravitation and Cosmology, (Wiley, New York, 1972).

[11] J. W. Cronin, Nucl. Phys. B (Proc. Suppl.) 28B, 213 (1993).

[12] Pair production can be neglected for propagation distances less than $100 \mathrm{Mpc}$ even in the case of heavy nuclei. G. Blumenthal, Phys. Rev. D1, 1596 (1970).

[13] F. W. Stecker, Phys. Rev D 180, 1264 (1969). An equivalent expression was presented by Berezinsky and Zatsepin in, V. S. Berezinsky and G. T. Zatsepin, Yad. Fiz. 13, 797 (1971) [Sov. J. Nucl. Phys. 13, 453 (1971)].

[14] J. L. Puget, F. W. Stecker and J. H. Bredekamp, Ap. J. 205, 638 (1976).

[15] L. A. Anchordoqui, M. T. Dova, L. Epele and J. Swain, Proc. 25 $5^{\text {th }}$ International Cosmic Ray Conference, Durban, South Africa, 1997, edited by M. S. Potgieter, B. C. Raubenheimer and D. J. van der Walt, Vol. 7, p.353.

[16] When Lorentz factors are less than $2 \times 10^{9}$, photonuclear interactions of the infrared background radiation become important. Iron nuclei of $\approx 200 \mathrm{EeV}$ can propagate $\approx$ $100 \mathrm{Mpc}$. The reader is refered to, F. W. Stecker, astro$\mathrm{ph} / 9710353$.

[17] L. A. Anchordoqui, M. T. Dova, L. Epele and J. Swain, Phys. Rev. D55, 7356 (1997).

[18] The Auger Collaboration, Pierre Auger Project Design Report, 1995, Fermi National Accelerator Laboratory (unpublished). 